\title{
A new species of Polypedilum (Cerobregma) (Diptera, Chironomidae) from Oriental China
}

\author{
Wen-Bin Liu', Yuan Yao', Chun-Cai Yan', Xin-Hua Wang', Xiao-Long Lin² \\ I Tianjin Key Laboratory of Conservation and Utilization of Animal Diversity, Tianjin Normal University, \\ Tianjin, 300387, China 2 College of Life Sciences, Nankai University, Tianjin, 300071, China \\ Corresponding author: Xiao-Long Lin (lin880224@gmail.com)
}

Academic editor: F.L. da Silva | Received 11 October 2020 | Accepted 15 December 2020 | Published 22 January 2021

http://zoobank.org/B350C4DE-6597-499C-B209-D9A363A6F88D

Citation: Liu W-B, Yao Y, Yan C-C, Wang X-H, Lin X-L (2021) A new species of Polypedilum (Cerobregma) (Diptera, Chironomidae) from Oriental China.. ZooKeys 1011: 139-148. https://doi.org/10.3897/zookeys.1011.59554

\begin{abstract}
Polypedilum (Cerobregma) huapingensis Liu \& Lin, sp. nov. is described and illustrated based on an adult male from Huaping National Nature Reserve, Guangxi, China. A DNA barcode analysis, including the known partial COI sequences of species in the Cerobregma subgenus, was conducted. An updated key to adult males of the subgenus Cerobregma is provided.
\end{abstract}

\section{Keywords}

COI, Chironomini, integrative taxonomy, new species

\section{Introduction}

The genus Polypedilum Kieffer is one of the largest chironomid genera, containing eight subgenera and more than 520 described species (Sæther et al. 2010; Cranston et al. 2016; Yamamoto et al. 2016; P. Ashe, pers. comm.). The larvae mostly occur in sediments, but some species are associated with mines of aquatic plants or co-inhabit pupal retreats of caddisflies (Cranston et al. 1989). Adult males of the subgenus Cerobregma Sxther \& Sundal, 1999 are characterized by having extremely long and strong, split setae along the inner margin of the gonostylus and gonocoxite, with an apicolateral 
bulb-like extension with deep lateral incision between the bulb and the gonostylus. The subgenus Cerobregma includes 15 valid species recorded in the Afrotropical, Holarctic and Oriental regions (Tokunaga 1940; Sxther and Sundal 1999; Kobayashi et al. 2003; Zhang and Wang 2005; Zhang et al. 2006; Moubayed-Breil 2007; Tang and Niitsuma 2017; Lin et al. 2019).

The DNA barcode corresponding to the 658-bp fragment of the mitochondrial gene cytochrome c oxidase I (COI) has been identified as the core of a global bio-identification system at the species level (Hebert et al. 2003a, b) and has proved to be useful in non-biting midge species delimitation (Anderson et al. 2013; Silva et al. 2014; Lin et al. 2015; Montagna et al. 2016; Giłka et al. 2018; Lin et al. 2018; Qi et al. 2017). COI barcodes have provided important evidence to confirm new species descriptions within Polypedilum species (Song et al. 2016, 2018; Lin et al. 2019).

The Nanling-Mountain region, located in the middle subtropical zone of China, rich in biological resources and with a warm and moist climate, is a typical natural ecosystem and one of the most biologically diverse areas in the world. Recently, during investigations of insect diversity in the Nanling Mountains, we discovered an unknown species of the subgenus Cerobregma from Huaping National Nature Reserve. In the present study, Polypedilum (Cerobregma) huapingensis Liu \& Lin sp. nov. is described and delimited by its morphology and DNA barcode. An updated key to adult males of the subgenus is provided.

\section{Materials and methods}

The single specimen of the new species, collected by a Malaise trap, was preserved in $85 \%$ ethanol and stored in the dark at $4^{\circ} \mathrm{C}$ before morphological and molecular analyses. Genomic DNA was extracted from the thorax and head using a Qiagen DNA Blood and Tissue Kit at Nankai University, Tianjin, China, following the standard protocol except for the final elution volume of $100 \mu \mathrm{l}$. After DNA extraction, the exoskeleton of each specimen was mounted in Euparal on a microscope slide together with the corresponding wings, legs, antennae and abdomen, following the procedures outlined by Sæther (1969). Morphological terminology follows Sæther (1980).

The color pattern of new species is described based on the specimen preserved in ethanol. Digital photographs of slide-mounted specimens were taken with a 300-dpi resolution using a Nikon Digital Sight DS-Fil camera mounted on Nikon Eclipse 80i compound microscope using the software NIS-Elements F v.4.60.00. at the College of Life Sciences, Nankai University, Tianjin, China.

The universal primers LCO1490 and HCO2198 (Folmer et al. 1994) were used to amplify the standard 658-bp mitochondrial COI barcode region. Polymerase chain reaction (PCR) amplifications followed Song et al. (2018) and were conducted in a $25 \mu \mathrm{l}$ volume including $12.5 \mu \mathrm{l} 2 \times$ Es Taq MasterMix (CoWin Biotech Co., Beijing, China), $0.625 \mu \mathrm{l}$ of each primer, $2 \mu \mathrm{l}$ of template DNA and $9.25 \mu \mathrm{l}$ of deionized $\mathrm{H}_{2} \mathrm{O}$. 
PCR products were electrophoresed in 1.0\% agarose gel, and purified and sequenced in both directions at Beijing Genomics Institute Co. Ltd., Beijing, China.

Raw sequences were assembled and edited in Geneious Prime 2020 (Biomatters Ltd., Auckland, New Zealand). Alignment of the sequences was carried out using the MUSCLE algorithm (Edgar 2004) on amino acids in MEGA 6 (Tamura et al. 2013). The pairwise distances using the Kimura 2-Parameter (K2P) substitution model of six species within the subgenus Cerobregma were calculated in MEGA. The neighborjoining tree was constructed using the K2P substitution model, 1000 bootstrap replicates and the "pairwise deletion" option for missing data in MEGA. Novel sequences, trace-files, and metadata of the new species were uploaded to the Barcode of Life Data Systems (BOLD) (Ratnasingham and Hebert 2013). The GenBank accession number for the new species is MW472357.

The holotype of the new species is deposited at the College of Life Sciences, Nankai University, Tianjin, China (NKU).

\section{Results}

\section{DNA barcode analysis}

The neighbor joining tree based on COI DNA barcodes of the six sequenced species within the subgenus Cerobregma revealed six distinct genetic clusters, suggesting one species new to science (Fig. 1). In some barcode studies in Chironomidae, the average threshold intraspecific divergence is $4-5 \%$ in Tanytarsus van der Wulp (Lin et al. 2015) and 5\%-8\% in Polypedilum Kieffer (Song et al. 2016, 2018). Polypedilum (Cerobregma) huapingensis sp. nov. can be differentiated from the other sequenced species by a more than 13\% divergence in the COI barcode sequence (Tab. 1; Fig. 1).

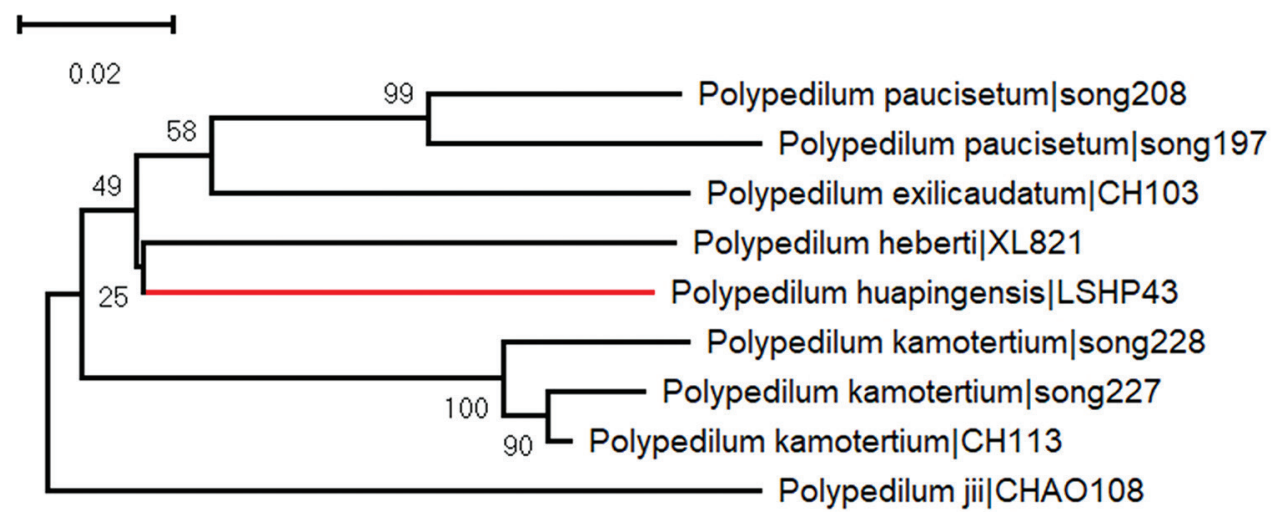

Figure I. Neighbor-joining tree for six species of the subgenus Cerobregma based on K2P distance in DNA barcodes. Numbers on branches represent bootstrap support (>70\%) based on 1000 replicates; scale equals K2P genetic distance. 
Table I. Kimura 2-parameter pairwise genetic distances based on COI barcodes of the Polypedilum (Cerobregma).

\begin{tabular}{|c|c|c|c|c|c|c|c|c|c|}
\hline Species & Specimen reference number & & & Pairwise gene & etic dist & tances & & & GenBank accessions \\
\hline P. exilicaudatum & $\mathrm{CH} 103$ & & & & & & & & MG950021 \\
\hline P. yamasinense & song227 & 0.155 & & & & & & & MG949754 \\
\hline P. yamasinense & $\mathrm{CH} 113$ & 0.147 & 0.015 & & & & & & MG949955 \\
\hline P. jii & CHAO108 & 0.185 & 0.168 & 0.156 & & & & & MG950056 \\
\hline P. yamasinense & song228 & 0.155 & 0.041 & $0.034 \quad 0.179$ & & & & & MG950029 \\
\hline P. paucisetum & song208 & 0.117 & 0.161 & $0.147 \quad 0.171$ & 0.157 & & & & MG950008 \\
\hline P. paucisetum & song197 & 0.138 & 0.163 & $0.153 \quad 0.162$ & 0.170 & 0.075 & & & MG949790 \\
\hline P. heberti & XL821 & 0.140 & 0.146 & $0.137 \quad 0.174$ & 0.157 & 0.142 & 0.155 & & MK505566 \\
\hline P. huapingensis & LSHP43 & 0.130 & 0.145 & $0.136 \quad 0.173$ & 0.153 & 0.140 & 0.159 & 0.135 & MW472357 \\
\hline
\end{tabular}

\section{Taxonomy}

\section{Polypedilum (Cerobregma) huapingensis Liu \& Lin, sp. nov.} http://zoobank.org/79EB5D46-B309-4459-8D2C-903589EC65F3

Figures 2-4

Type material. Holotype: male (NKU \& BOLD sample ID: LSHP43), China, Guangxi Zhuang Autonomous Region, Guilin City, Lingui County, Huaping National Nature Reserve, $25.563^{\circ} \mathrm{N}, 109.942^{\circ} \mathrm{E}, 1271 \mathrm{~m}$ a.s.l., $10-20 . V I .2020$, Malaise trap, S.G. Zhao.

Etymology. The specific name refers to the Huaping National Nature Reserve, where the holotype was collected.

Diagnostic characters. According to the morphological characters of the adult male, the new Polypedilum species keys to the subgenus Cerobregma, and can be distinguished from other known species of the subgenus by the following combination of characters: tergites III-VI brown with dark brown spots at middle; wing pale brown with a large black spot on entire basal area of wing; superior volsella with basal microtrichia and two inner setae; anal point strong, contracted in middle, a large inflated globe apically with candle-like spine.

Adult male $(\mathbf{n}=\mathbf{1})$. Total length $4.29 \mathrm{~mm}$. Wing length $2.71 \mathrm{~mm}$. Total length/ wing length 1.58. Wing length/length of profemur 1.57.

Coloration (Fig. 2). Head brown. Antenna yellow. Thorax ground color brown with dark brown stripes on scutum, laterally under parapsidal suture, postnotum and on preepisternum. Tergites III-VI brown with dark brown spots at middle; tergites I, II, VII, and VIII and hypopygium largely dark brown. Most of femora and tibiae dark brown, all tarsomeres yellow. Wing pale brown with a large black spot on entire basal area.

Head (Fig. 3A). Antenna with 13 flagellomeres; ultimate flagellomere $425 \mu \mathrm{m}$ long; AR 0.44 . Temporal setae 27, including 7 inner verticals and 20 outer verticals. Clypeus with 79 setae. Tentorium $132 \mu \mathrm{m}$ long; $45 \mu \mathrm{m}$ wide. Stipes $112 \mu \mathrm{m}$ long, $24 \mu \mathrm{m}$ wide. Lengths of palpomeres 1-5 (in $\mu \mathrm{m}$ ): 52.5, 97.5, 192, 170, 232. Palpomere ratio $\left(5^{\text {th }} / 3^{\text {rd }}\right) 1.21$.

Thorax (Fig. 3B). Acrostichals 31; humerals 38; dorsocentrals 104; prealars 16. Scutellum with 86 setae. 


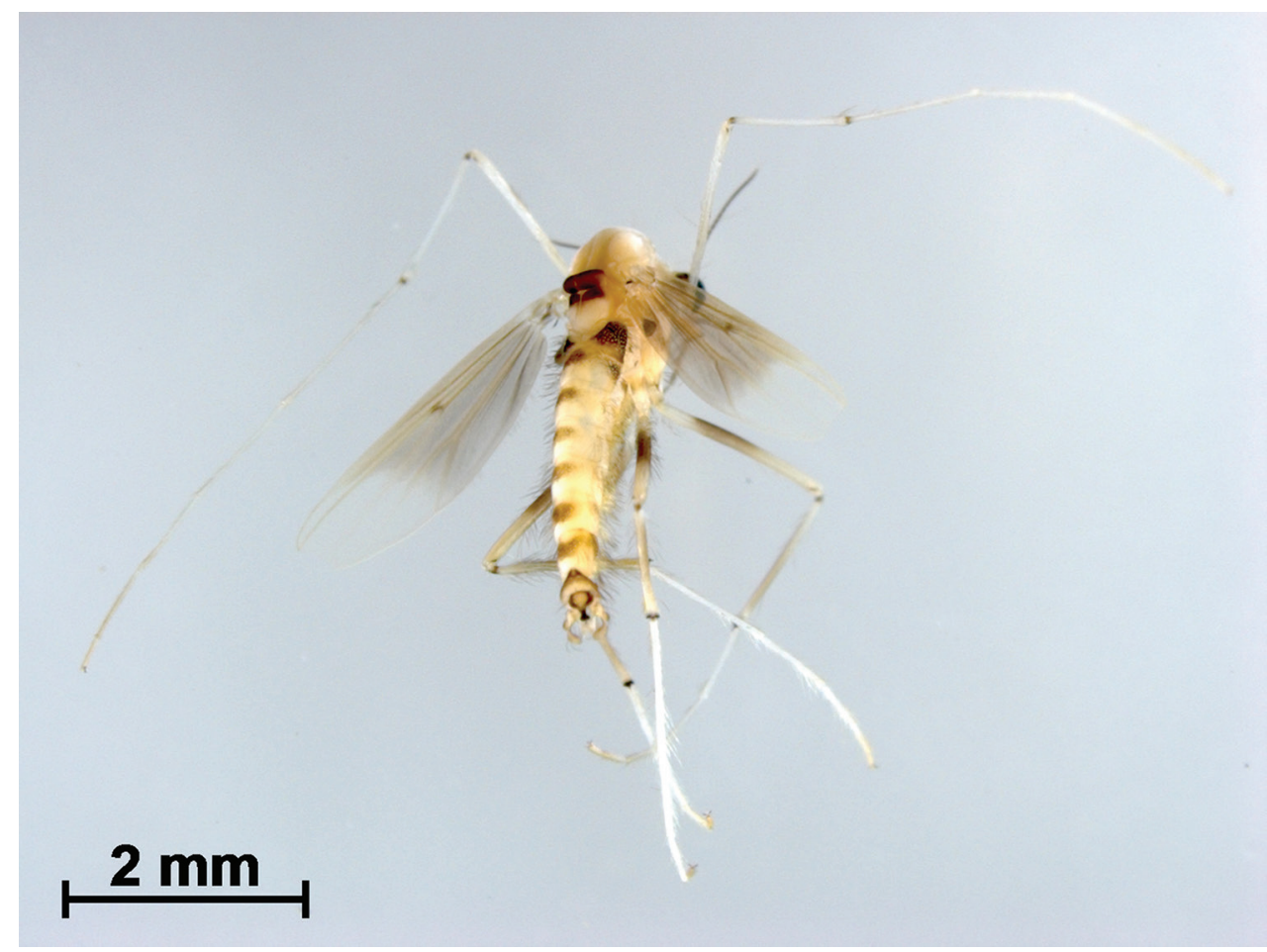

Figure 2. Polypedilum (Cerobregma) huapingensis Liu \& Lin, sp. nov., holotype male.

Wing (Fig. 3C). VR 1.15. Brachiolum with 13 setae; $\mathrm{R}$ with 36 setae; $\mathrm{R}_{1}$ with 32 setae; $\mathrm{R}_{4+5}$ with 36 setae; $M$ with 22 setae; remaining veins bare. $\mathrm{R}_{2+3}$ distinct. Squama with 16 setae. Anal lobe moderately developed.

Legs. Spur of mid tibia $28 \mu \mathrm{m}$ long, mid tibia including $55 \mu \mathrm{m}$ long comb, unspurs comb $35 \mu \mathrm{m}$ long; spur of hind tibia $36 \mu \mathrm{m}$ long including $78 \mu \mathrm{m}$ long comb, un-spurs comb $40 \mu \mathrm{m}$ long. Apical width of fore tibia $60 \mu \mathrm{m}$; of mid tibia $76 \mu \mathrm{m}$; of hind tibia $80 \mu \mathrm{m}$. Lengths (in $\mu \mathrm{m}$ ) and proportions of legs as in Table 2.

Hypopygium (Fig. 4). Anal tergite with 46 median setae. Laterosternite IX with seven setae. Anal point as in Fig. 4D, strong, contracted in middle, as a large inflated globe apically with a single candle-like spine, tapering, $143 \mu \mathrm{m}$ long. Transverse sternapodeme $93 \mu \mathrm{m}$ long; phallapodeme $168 \mu \mathrm{m}$ long. Gonocoxite $320 \mu \mathrm{m}$ long. Superior volsella $143 \mu \mathrm{m}$ long, with basal microtrichia and two inner setae (Fig. 4E). Inferior

Table 2. Lengths (in $\mu \mathrm{m}$ ) and proportions of legs of Polypedilum (Cerobregma) huapingensis sp. nov., male holotype $(n=1)$.

\begin{tabular}{lccccccccccc}
\hline & $\mathbf{f e}$ & $\mathbf{t i}$ & $\mathbf{t a}_{\mathbf{1}}$ & $\mathbf{t a}_{\mathbf{2}}$ & $\mathbf{t a}_{\mathbf{3}}$ & $\mathbf{t a}_{\mathbf{4}}$ & $\mathbf{t a}_{\mathbf{5}}$ & $\mathbf{L R}$ & $\mathbf{B V}$ & $\mathbf{S V}$ & $\mathbf{B R}$ \\
\hline $\mathrm{P}_{1}$ & 1725 & 1108 & 2405 & 1025 & 725 & 625 & 275 & 2.17 & 2.11 & 1.98 & 3.14 \\
$\mathrm{P}_{2}$ & 1702 & 1225 & 655 & 475 & 355 & 175 & 148 & 0.53 & 2.67 & 3.11 & 4.50 \\
$\mathrm{P}_{3}$ & 1850 & 1208 & 1075 & 925 & 503 & 362 & 154 & 0.89 & 2.34 & 2.13 & 4.90 \\
\hline
\end{tabular}



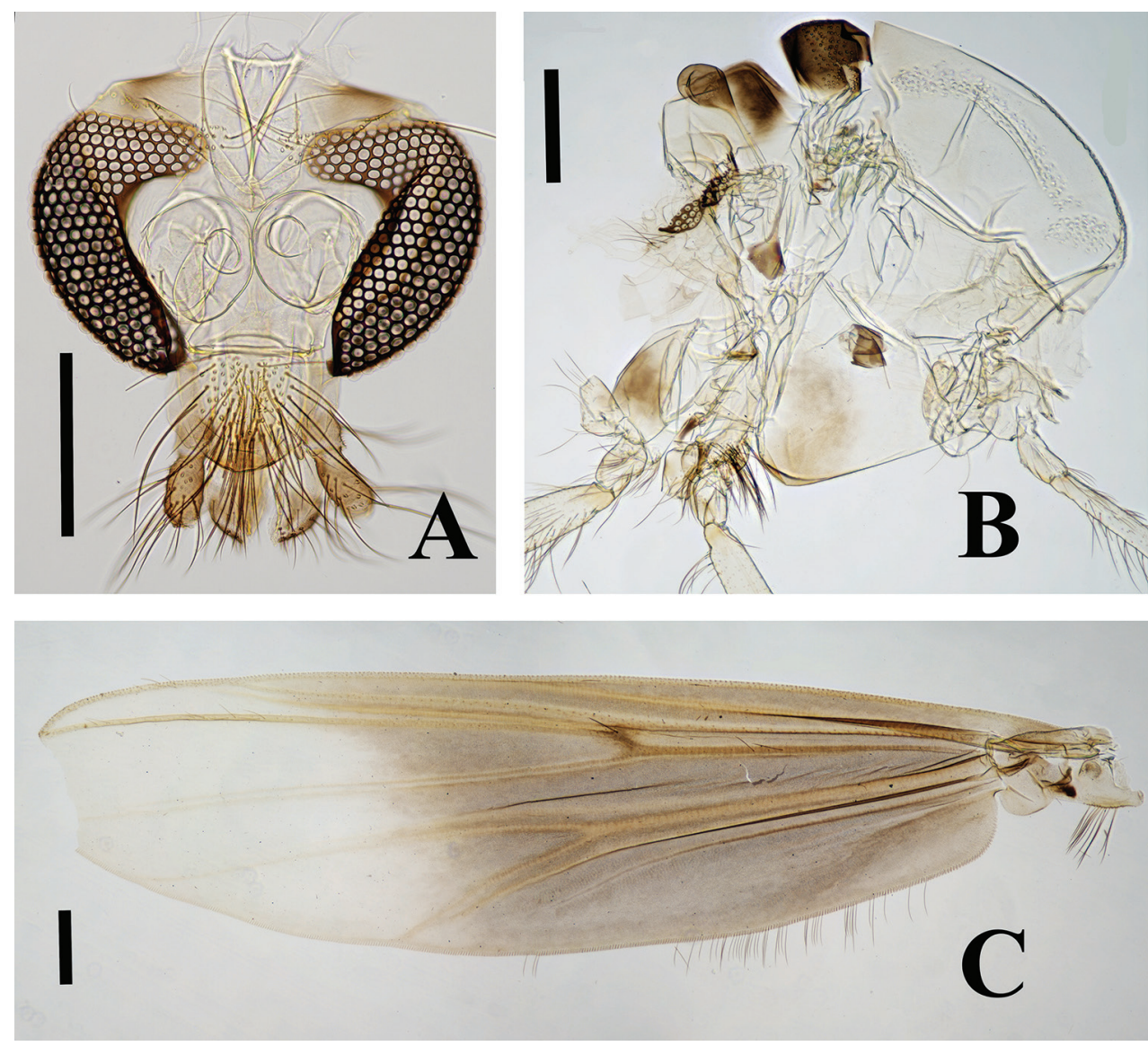

Figure 3. Polypedilum (Cerobregma) huapingensis Liu \& Lin, sp. nov., holotype male A head B thorax C wing. Scale bars: $200 \mu \mathrm{m}$.

volsella $164 \mu \mathrm{m}$ long, finger-shaped, divided into two lobes apically, with 24 long setae. Gonostylus $310 \mu$ m long. HR 1.03; HV 1.38.

Female and immatures unknown.

Discussion. The characters of the anal point and superior volsella of the new species place it within the subgenus Cerobregma. The morphology of the new species resembles that of Polypedilum heberti Lin \&Wang, 2019, but it can be separated from it on the basis of the following: 1) tergites III-VI brown with dark brown spots at middle in the new species, versus tergites III-VI with dark brown bands at middle in $P$. heberti; 2) thorax of the new species (acrostichals 31; humerals 38; dorsocentrals 104) with much more setae than in $P$. heberti (acrostichals 8; humerals 5; dorsocentrals 20); 3 ) anal point strong and tapering in $P$. heberti, versus constricted in middle, with a large inflated globe apically with candle-like spine in the new species. 

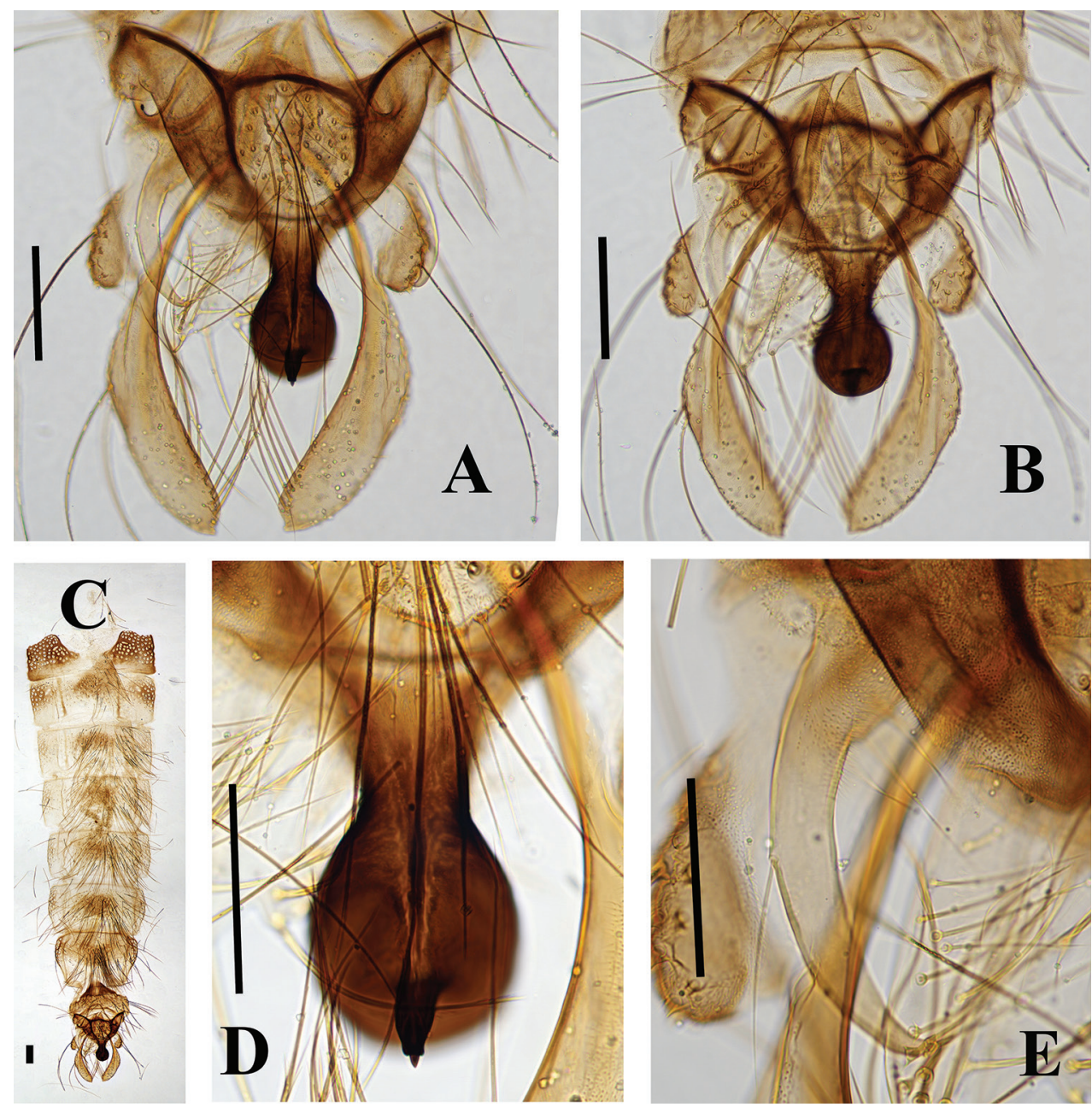

Figure 4. Polypedilum (Cerobregma) huapingensis Liu \& Lin, sp. nov., holotype male A hypopygium, dorsal view B hypopygium, ventral view $\mathbf{C}$ abdomen $\mathbf{D}$ superior volsella $\mathbf{E}$ anal point. Scale bars: $100 \mu \mathrm{m}$.

\section{Updated key to known adult males of Polypedilum (Cerobregma)}

The following key replaces couplet 5 in Lin et al. (2019) and adds a couplet 5a to include the male of the newly described species.

$5 \quad$ Wing with several spots; setae along inner margin of gonostylus strongly split

P. ramiferum Kieffer, 1921

- Wing with a large black spot on entire basal area; setae along inner margin of gonostylus not split 
5a Acrostichals 8; humerals 5; dorsocentrals 20; anal point strong and tapering... P. heberti Lin \& Wang, 2019

- $\quad$ Acrostichals 31; humerals 38; dorsocentrals 104; anal point strong, contracted in middle, as a large inflated globe apically with candle-like spine

P. huapingensis Liu \& Lin, sp. nov.

\section{Acknowledgements}

Financial support from the National Natural Science Foundation of China (31672264, 31672324, 31801994, 31900344), the China Postdoctoral Science Foundation Grant (2018M640227), GDAS Special Project of Science and Technology Development (No. 2020GDASYL-20200102021, 2020GDASYL-20200301003) and the Natural Science Foundation of Tianjin (18JCQNJC14700, 18JCYBJC96100, S20QNS624) is acknowledged with thanks.

\section{References}

Anderson AM, Stur E, Ekrem T (2013) Molecular and morphological methods reveal cryptic diversity and three new species of Nearctic Micropsectra (Diptera: Chironomidae). Freshwater Science 32: 892-921. https://doi.org/10.1899/12-026.1

Cranston PS, Dillon ME, Pinder LCV, Reiss F (1989) The adult males of Chironominae (Diptera: Chironomidae) of the Holarctic region - Keys and diagnoses. In: Wiederholm T (Ed.) Chironomidae of the Holarctic region. Keys and diagnoses. Part 3 - Adult males. Entomologica Scandinavica Supplement 34: 353-502.

Cranston PS, Martin J, Spies M (2016) Cryptic species in the nuisance midge Polypedilum nubifer (Skuse (Diptera: Chironomidae) and the status of Tripedilum Kieffer. Zootaxa 4079: 429-447. https://doi.org/10.11646/zootaxa.4079.4.3

Edgar RC (2004) MUSCLE: multiple sequence alignment with high accuracy and high throughput. Nucleic Acids Research 32: 1792-1797. https://doi.org/10.1093/nar/gkh340

Folmer O, Black M, Hoeh W, Lutz R, Vrijenhoek R (1994) DNA primers for amplification of mitochondrial cytochrome $c$ oxidase subunit I from diverse metazoan invertebrates. Molecular Marine Biology and Biotechnology 3: 294-299.

Giłka W, Paasivirta L, Gadawski P, Grabowski M (2018) Morphology and molecules say: Tanytarsus latens, sp. nov. from Finland (Diptera: Chironomidae). Zootaxa 4471: 569-579. https://doi.org/10.11646/zootaxa.4471.3.8

Hebert PDN, Cywinska A, Ball SL (2003a) Biological identifications through DNA barcodes. Proceedings of the Royal Society of London B: Biological Sciences 270: 313-321. https:// doi.org/10.1098/rspb.2002.2218

Hebert PDN, Ratnasingham S, de Waard JR (2003b) Barcoding animal life: cytochrome $c$ oxidase subunit 1 divergences among closely related species. Proceedings of the Royal Society of London B: Biological Sciences 270: S96-S99. https://doi.org/10.1098/rsbl.2003.0025 
Kobayashi T, Ohtaka A, Takahashi T (2003) The second record of ectoparasitic Chironomidae on Trichoptera from Japan, Polypedilum (Cerobregma) kamotertium Sasa, 1989 (Insecta, Diptera, Chironomidae, Chironomini). Spixiana 26: 83-91.

Lin XL, Stur E, Ekrem T (2015) Exploring genetic divergence in a species-rich insect genus using 2790 DNA Barcodes. PLoS ONE 10: e0138993. https://doi.org/10.1371/journal. pone. 0138993

Lin XL, Stur E, Ekrem T (2018) DNA barcodes and morphology reveal unrecognized species of Chironomidae (Diptera). Insect Systematics \& Evolution 49: 329-398. https://doi. org/10.1163/1876312X-00002172

Lin XL, Yu HJ, Zhang RL, Wang XH (2019) Polypedilum (Cerobregma) heberti sp. n. (Diptera: Chironomidae) from Gaoligong Mountains, Yunnan, China. Zootaxa 4571(2): 255-262. https://doi.org/10.11646/zootaxa.4571.2.5

Montagna M, Mereghetti V, Lencioni V, Rossaro B (2016) Integrated taxonomy and DNA barcoding of alpine midges (Diptera: Chironomidae). PLoS ONE 11(3): e0149673. https:// doi.org/10.1371/journal.pone.0149673

Moubayed-Breil J (2007) Polypedilum (Cerobregma) lotensis, new species, and P. (C.) saetheri, new species, from lowland streams and rivers in France (Diptera: Chironomidae). In: Andersen T (Ed.) Contributions to the systematics and ecology of aquatic Diptera-A tribute to Ole A. Sæther. The Caddis Press, Columbus, Ohio, 205-213.

Qi X, Wang XH, Andersen T, Lin XL (2017) A new species of Manoa Fittkau (Diptera: Chironomidae), with DNA barcodes from Xianju National Park, Oriental China. Zootaxa 4231: 398-408. https://doi.org/10.11646/zootaxa.4231.3.6

Ratnasingham S, Hebert PDN (2013) A DNA-based registry for all animal species: the barcode index number (BIN) system. PLoS ONE 8: e66213. https://doi.org/10.1371/journal.pone.0066213

Sæther OA (1969) Some Nearctic Podonominae, Diamesinae, and Orthocladiinae (Diptera: Chironomidae). Bulletin of the Fisheries Research Board of Canada 170: 1-154.

Sæther OA (1980) Glossary of chironomid morphology terminology (Diptera: Chironomidae). Entomologica Scandinavica Supplement 14: 1-51.

Sæther OA, Andersen T, Pinho LC, Mendes HF (2010) The problems with Polypedilum Kieffer (Diptera: Chironomidae), with the description of Probolum subgen. n. Zootaxa 2497: 1-36. https://doi.org/10.11646/zootaxa.2497.1.1

Sæther OA, Sundal A (1999) Cerobregma, a new subgenus of Polypedilum Kieffer, with a tentative phylogeny of subgenera and species groups within Polypedilum (Diptera: Chironomidae). Journal of the Kansas Entomological Society 71: 315-382.

Silva FL, Fonseca-Gessner AA, Ekrem T (2014) A taxonomic revision of genus Labrundinia Fittkau, 1962 (Diptera: Chironomidae: Tanypodinae). Zootaxa 3769(1): e185. https:// doi.org/10.11646/zootaxa.3769.1.1

Song C, Lin XL, Wang Q, Wang XH (2018) DNA barcodes successfully delimit morphospecies in a superdiverse insect genus. Zoologica Scripta 47: 311-324. https://doi.org/10.1111/zsc.12284

Song C, Wang Q, Zhang RL, Sun BJ, Wang XH (2016) Exploring the utility of DNA barcoding in species delimitation of Polypedilum (Tripodura) non-biting midges (Diptera: Chironomidae). Zootaxa 4079: 534-550. https://doi.org/10.11646/zootaxa.4079.5.2 
Tamura K, Stecher G, Peterson D, Filipski A, Kumar S (2013) MEGA6: molecular evolutionary genetics analysis version 6.0. Molecular Biology and Evolution 30: 2725-2729. https:// doi.org/10.1093/molbev/mst197

Tang HQ, Niitsuma H (2017) Review of the Japanese Microtendipes (Diptera: Chironomidae), with description of a new species. Zootaxa 4320: 535-553. https://doi.org/10.11646/ zootaxa.4320.3.8

Tokunaga M (1940) Chironomidae from Japan (Diptera), XI1. New or little-known Ceratopogonidae and Chironomidae. The Philippine Journal of Science 72: 255-311.

Yamamoto N, Yamamoto M, Hirowatari T (2016) Erection of a new subgenus in the genus Polypedilum Kieffer, 1912 (Diptera: Chironomidae) from Japan. Japanese Journal of Systematic Entomology 22: 195-197.

Zhang RL, Wang XH (2005) Polypedilum (Cerobregma) Sæther \& Sundal from China (Diptera: Chironomidae). Aquatic Insects 27: 47-55. https://doi.org/10.1080/01650420400019262

Zhang RL, Wang XH, Sæther OA (2006) Two unusual species of Polypedilum Kieffer (Diptera: Chironomidae) from Oriental China. Zootaxa 1282: 39-48. https://doi.org/10.11646/ zootaxa.1282.1.4 